\title{
On the problem of possibilistic-probabilistic optimization with constraints on possibility/probability in the case of the weakest t-norm
}

\author{
Egorova Yu.E. ${ }^{a}$ and Yazenin A.V. ${ }^{b}$ \\ ${ }^{a}$ Department of Information Technology, Faculty of Computational Mathematics and Cybernetics, \\ Tver State University, 33 Zhelyabova Str., Tver, 170000, j.e.egorova@gmail.com \\ ${ }^{b}$ Department of Information Technology, Faculty of Computational Mathematics and Cybernetics, \\ Tver State University, 33 Zhelyabova Str., Tver, 170000, yazenin.av@tversu.ru
}

\begin{abstract}
In this paper we investigate the problem of possibilistic-probabilistic optimization with constraints on possiblity/probability. Fuzzy parameters of the problem are considered to be related by the weakest t-norm, random parameters are normally distributed. For solving the problem an equivalent stochastic problem is constructed.
\end{abstract}

Keywords: Possibilistic-probabilistic optimization, possibility/probability constraints, the weakest t-norm, stochastic quasigradient method

\section{Introduction}

In the real world making a decision is often complicated by the vagueness of the information. To deal with such decision making problems, methods of fuzzy random optimization has been extensively developed.

In works $[1,12,13,14,15]$ models of fuzzy random optimization were presented and various methods for their solving were discussed. In [9] models and methods of fuzzy random optimization were developed for multi-objective optimization. Nowadays, most researches are based on the notions of fuzzy random variable introduced by $H$. Kwakernaak $[10,11]$ and M. Puri and D. Ralescu [18],

In contrast to these works, we base our studies on the notion of fuzzy random (possibilistic-probabilistic) variable that has been introduced by Nahmias [16]. In $[19,20]$ the models of possibilitic-probabilistic optimization were defined and methods to solve them were studied. These methods use the principle of expected possibility to construct an equivalent problem. The construction consists of two steps: elimination of probabilistic (de-randomization) and possibilitic (defuzzification) uncertainties. De-randomization is per- formed by using the expected value, whrereas defuzzification is performed by applying requirements to the degree of possibility/necessity of satisfying the critera and constraints of the problem. The works $[4,24]$ continue the studies in this field.

In this paper we investigate the model studied in [25], where for the elimination of the probabilistic uncertainty probability constraints are used.

In contrast to [25], we consider that the interaction of fuzzy paramteres are described by the weakest (drastic) t-norm $T_{W}$. Since any t-norm is pointwise bounded by the drastic t-norm and the minimum tnorm, this will allow us to estimate the range of the criteria function and also the behavior of the feasible solution domain, thereby managing the uncertainty in the course of decision-making.

For solving the problem studied in this paper, an equivalent crisp problem can be constructed. However, obtaining equivalent crisp problems requires the calculation of the precise values of the expected value and the variance of a random function. In the case of the weakest t-norm the expected value and the variance of a a fuzzy random function are of such a complex nature that the calculation of their precise values is almost impossible. Moreover, the results of this calculation are nonlinear non-convex functions, which lead to a non-convex optimization problem.

To avoid difficult calculations we construct an equivalent stochastic problem and propose methods of stochastic programming, such as the stochastic quasigradient methods, to solve it.

\section{Materials and Methods}

We introduce a number of definitions and concepts from the possibility theory following [16, 22]. Let $(\Gamma, \mathrm{P}(\Gamma), \tau)$ and $(\Omega, \mathrm{B}, P)$ be possibility and probability spaces where $\Omega$ is a sample space with possible outcomes $\omega \in \Omega, \Gamma$ is a pattern space with elements 
$\gamma \in \Gamma, \mathrm{B}$ is an $\sigma$-algebra of events, $\mathrm{P}(\Gamma)$ is the discrete topology on $\Gamma, \tau=(\pi, \nu), \pi$ and $\nu$ are measures of possibility and necessity respectively, $P$ is a probability measure and $\mathrm{E}^{1}$ is the real line.

Definition 1. Fuzzy random variable $Y$ is a real function $Y: \Omega \times \Gamma \rightarrow \mathrm{E}^{1}$, which is $\sigma$-measurable for each fixed $\gamma$ and

$$
\mu_{Y}(\omega, t)=\pi\{\gamma \in \Gamma: Y(\omega, \gamma)=t\}
$$

is called its distribution function.

It follows from Definition 1 that the distribution function of a fuzzy random variable depends on a random parameter, that is, it is a random function.

Definition 2. Let $Y(\omega, \gamma)$ be a fuzzy random variable. Its expected value $\mathrm{E}[Y]$ is a fuzzy variable with the possibility distribution function

$$
\mu_{\mathrm{E}[Y]}(t)=\pi\{\gamma \in \Gamma: \mathrm{E}[Y(\omega, \gamma)]=t\}
$$

where $\mathrm{E}$ is the mathematical expectation operator

$$
\mathrm{E}[Y(\omega, \gamma)]=\int_{\Omega} Y(\omega, \gamma) P(\mathrm{~d} \omega)
$$

In this case, the distribution function of the expected value of a fuzzy random variable is no longer dependent on a random parameter and is therefore deterministic.

The most interesting representation of a fuzzy random variable is a shift-scale representation [22]:

$$
Y(\omega, \gamma)=a(\omega)+\sigma(\omega) Z(\gamma)
$$

where $a(\omega), \sigma(\omega)$ are random variables defined on the probability space $(\Omega, \mathrm{B}, P)$ and $Z(\gamma)$ is a fuzzy variable defined on the possibility space $(\Gamma, \mathrm{P}(\Gamma), \tau)$. Random components $a(\omega)$ and $\sigma(\omega)$ are called the shift and the scale respectively.

For a better intuitive understanding of such a representation of a fuzzy random variable imagine a situation where some financial expert is asked to estimate a return of a certain financial asset. Both the return and its estimation by the expert are uncertain quantities. We assume that the uncertainty determined by market conditions has a probabilistic nature. On the other hand, uncertainty of the estimation by the expert is described by some possibility distribution. This model seems quite plausible if we assume that the degree of fuzziness of the expert depends mainly on the scale of variation of the estimated variable and not on its true value [22].

LR-type distributions are often used to model fuzzy numbers [3].
Definition 3. $Z(\gamma)$ is called an LR-type fuzzy variable if its distribution function has the form

$$
\mu_{Z}(t)= \begin{cases}L\left(\frac{\underline{m}-t}{\underline{d}}\right), & t<\underline{m}, \\ 1, & t \in[\underline{m}, \bar{m}], \\ R\left(\frac{t-\bar{m}}{\bar{d}}\right), & t>\bar{m},\end{cases}
$$

where $L(t), R(t)$ are shape functions.

In this case $Z(\gamma)$ is written in the form $Z=$ $[\underline{m}, \bar{m}, \underline{d}, \bar{d}]_{L R}$, where $\underline{m} \leq \bar{m}$ are the left and the right limits of tolerance (modal) interval, $\underline{d}>0, \bar{d}>0$ are coefficients of fuzziness.

A fuzzy variable $Z(\gamma)$ is called a symmetrical variable of LR-type, if $\underline{m}=\bar{m}$ and $\underline{d}=\bar{d}$ and $L(t)=R(t)$ for all $t \in E^{1}$. Its distribution can be written as $Z=$ $[\underline{m}, \underline{d}]_{L R}$.

We use triangular norms and conorms (t-norms and t-conorms) as an instrument for aggregation of fuzzy information. They generalize min and max operations on which actions on fuzzy sets and fuzzy variables are based [17].

In particular, in this work we consider two extreme t-norms

$$
T_{M}(x, y)=\min \{x, y\}
$$

and

$$
T_{W}(x, y)= \begin{cases}\min \{x, y\}, & \text { if } \max \{x, y\}=1 \\ 0, & \text { otherwise }\end{cases}
$$

$T_{M}$ and $T_{W}$ are called the strongest and the weakest t-norm respectively, since for any arbitrary t-norm $T$ and $\forall x, y \in[0,1]$ the following inequality holds [17]:

$$
T_{W}(x, y) \leq T(x, y) \leq T_{M}(x, y) .
$$

One of the main properties of t-norms is their ability to control uncertainty ("fuzziness") growth. The growth of fuzziness can appear, for example, when performing arithmetic operations on fuzzy numbers: if two fuzzy numbers of LR-type are summed using the strongest t-norm $T_{M}$, corresponding coefficients of fuzziness are also summed, therefore uncertainty is growing. With the help of t-norms other than $T$ we can slow the growth of fuzziness. The extreme triangular norms, which are considered in this work, give us boundaries for control of fuzziness in our optimization models.

Following [8] we introduce the notion of mutual Trelatedness of fuzzy sets and fuzzy variables. It is used as an instrument for construction of joint possibility distribution functions.

Definition 4. Fuzzy sets $A_{1}, \cdots, A_{n} \in \mathrm{P}(\Gamma)$ are called mutually T-related, if for any index set 


$$
\begin{aligned}
& \left\{i_{1}, \cdots, i_{k}\right\} \subset\{1, \cdots, n\}, k=\overline{1, n}, \text { we have } \\
& \quad \pi\left(A_{i_{1}} \cap \cdots \cap A_{i_{k}}\right)=T\left(\pi\left(A_{i_{1}}\right), \cdots, \pi\left(A_{i_{k}}\right)\right),
\end{aligned}
$$

where

$$
\begin{aligned}
& T\left(\pi\left(A_{i_{1}}\right), \cdots, \pi\left(A_{i_{k}}\right)\right)=T(T(\cdots \\
& \left.\left.T\left(T\left(\pi\left(A_{i_{1}}\right), \pi\left(A_{i_{2}}\right)\right), \pi\left(A_{i_{3}}\right)\right)\right), \pi\left(A_{i_{k}}\right)\right) .
\end{aligned}
$$

We can extend the notion of mutual T-relatedness of fuzzy sets to T-relatedness of fuzzy variables. Let $Z_{1}(\gamma), \cdots, Z_{n}(\gamma)$ be fuzzy variables defined on the possibility space $(\Gamma, \mathrm{P}(\Gamma), \pi)$.

Definition 5. Fuzzy variables $Z_{1}(\gamma), \cdots, Z_{n}(\gamma)$ are called mutually $T$-related if for any index set $\left\{i_{1}, \cdots, i_{k}\right\} \subset\{1, \cdots, n\}, k=\overline{1, n}$ and for for $t_{i_{j}} \in$ $\mathrm{E}^{1}$, we have

$$
\begin{aligned}
\mu_{Z_{i_{1}}, \cdots, Z_{i_{k}}} & \left(t_{i_{1}}, \cdots, t_{i_{k}}\right) \\
& =\pi\left\{\gamma \in \Gamma: Z_{i_{1}}(\gamma)=t_{i_{1}}, \cdots, Z_{i_{k}}(\gamma)=t_{i_{k}}\right\} \\
& =\pi\left\{Z_{i_{1}}^{-1}\left[t_{i_{1}}\right] \cap \cdots \cap Z_{i_{k}}^{-1}\left[t_{i_{k}}\right]\right\} \\
& =T\left\{\pi\left(Z_{i_{1}}^{-1}\left[t_{i_{1}}\right]\right), \cdots, \pi\left(Z_{i_{k}}^{-1}\left[t_{i_{k}}\right]\right)\right\} .
\end{aligned}
$$

\section{Possibilistic-Probabilistic Optimization Model}

In possibilistic-probabilistic optimization the principle of the expected possibility is mostly used. This principle allows to remove the probabilistic uncertainty by calculating of the expected values of possibilisticprobabilistic functions. In this paper we use probability constraints instead of the principle of the expected possibility. This approach was previously applied for portfolio analysis problems in $[5,21]$.

Consider a model of possibilistic-probabilistic optimization of the following form [24]:

$$
\begin{gathered}
k \rightarrow \min , \\
\pi\left\{P\left\{f_{0}(x, \omega, \gamma) \leq k\right\} \geq p_{0}\right\} \geq \alpha_{0}, \\
\left\{\begin{array}{l}
\pi\left\{P\left\{f_{i}(x, \omega, \gamma) \leq 0\right\} \geq p_{i}\right\} \geq \alpha_{i}, i=\overline{1, m} \\
x \in X .
\end{array}\right.
\end{gathered}
$$

Here $X \subseteq \mathrm{E}_{+}^{n}=\left\{x \in E^{n}: x \geq 0\right\} ; f_{i}(x, \omega, \gamma)$ are possibilistic-probabilistic functions

$$
f_{i}: X \times \Omega \times \Gamma \rightarrow E^{1}, i=\overline{0, m} ;
$$

$\alpha_{i} \in(0,1], k$ is a scalar variable.

Let $f_{i}(x, \omega, \gamma)$ for $i=\overline{0, m}$ be linear functions:

$$
f_{i}(x, \omega, \gamma)=\sum_{j=0}^{n} b_{i j}(\omega, \gamma) x_{j}-b_{i}(\omega, \gamma)
$$

with $b_{0}(\omega, \gamma)=0$. We assume that $b_{i j}(\omega, \gamma), b_{i}(\omega, \gamma)$ have a shift-scale representation

$$
\begin{gathered}
b_{i j}(\omega, \gamma)=a_{i j}(\omega)+\sigma_{i j}(\omega) Z_{i j}(\gamma), \\
b_{i}(\omega, \gamma)=a_{i}(\omega)+\sigma_{i}(\omega) Z_{i}(\gamma) .
\end{gathered}
$$

Here $a_{i j}(\omega), \sigma_{i j}(\omega)$ and $a_{i}(\omega), \sigma_{i}(\omega)$ are independent normally distributed random variables, the mathematical expectation of $\sigma_{i j}(\omega)$ and $\sigma_{i}(\omega)$ are positive; fuzzy components $Z_{i j}(\gamma)$ and $Z_{i}(\gamma)$ are represented by symmetrical fuzzy numbers of LR-type, i.e.

$$
Z_{i j}(\gamma)=\left[m_{i j}, d_{i j}\right]_{L R}, \quad Z_{i}(\gamma)=\left[m_{i}, d_{i}\right]_{L R},
$$

$L(t)=R(t), \forall t \geq 0$ for $i=\overline{0, m}$.

Let the interaction of the fuzzy parameters be described by the weakest t-norm $T_{W}$. Then the possibilistic distribution of the functions $f_{i}(x, \omega, \gamma)$ will be

$$
f_{i}(x, \omega, \gamma)=\left[m_{f_{i}}(x, \omega), d_{f_{i}}(x, \omega)\right]_{L R} .
$$

Here the functions $m_{f_{0}}(x, \omega)$ and $d_{f_{0}}(x, \omega)$ will have the following form

$$
\begin{gathered}
m_{f_{0}}(x, \omega)=\sum_{j=1}^{n}\left(a_{0 j}(\omega)+\sigma_{0 j}(\omega) m_{0 j}\right) x_{j}, \\
d_{f_{0}}(x, \omega)=\max \left\{\sigma_{01}(\omega) d_{01} x_{1}, \cdots, \sigma_{0 n}(\omega) d_{0 n} x_{n}\right\} .
\end{gathered}
$$

And for $i=\overline{1, m}$ the functions $m_{f_{i}}(x, \omega)$ and $d_{f_{i}}(x, \omega)$ will be

$$
\begin{gathered}
m_{f_{i}}(x, \omega)=\sum_{j=1}^{n}\left(a_{i j}(\omega)+\sigma_{i j}(\omega) m_{i j}\right) x_{j} \\
-\left(a_{i}(\omega)+\sigma_{i}(\omega) m_{i}\right), \\
d_{f_{i}}(x, \omega)=\max \left\{\sigma_{i 1}(\omega) d_{i 1} x_{1}, \cdots, \sigma_{i n}(\omega) d_{i n} x_{n},\right. \\
\left.\sigma_{i}(\omega) d_{i}\right\} .
\end{gathered}
$$

Note that for all $i=\overline{0, m}$ fuzzy random variables $f_{i}(x, \omega, \gamma)$ can also be represented in the following form

$$
f_{i}(x, \omega, \gamma)=m_{f_{i}}(x, \omega)+d_{f_{i}}(x, \omega) \cdot Z^{0}(\gamma),
$$

where $Z^{0}(\gamma)=[0,1]_{L R}$.

\section{Equivalent Stochastic Problem}

We now turn to the construction of an equivalent stochastic problem of the model (1)-(2). In order to do this, we need to transform (2) to an equivalent stochastic constraints model. After that the criteria can be reduced to an equivalent stochastic one in a similar way. 
Let $a_{i j}(\omega), \sigma_{i j}(\omega)$ and $a_{i}(\omega), \sigma_{i}(\omega)$ be normally distributed. Then for each $i=\overline{1, m}$ a constraint

$$
\pi\left\{P\left\{f_{i}(x, \omega, \gamma) \leq 0\right\} \geq p_{i}\right\} \geq \alpha_{i}
$$

can be transformed to [25]

$$
\pi\left\{\mathrm{E}\left[f_{i}(x, \omega, \gamma)\right]-\beta_{i} \sqrt{\mathrm{D}\left[f_{i}(x, \omega, \gamma)\right]} \leq 0\right\} \geq \alpha_{i},
$$

where $\mathrm{E}\left[f_{i}(x, \omega, \gamma)\right]$ is the mathematical expectation of the function $f_{i}(x, \omega, \gamma)$ and $\mathrm{D}\left[f_{i}(x, \omega, \gamma)\right]$ is its variance; $\beta_{i}$ is the solution of an equation

$$
F(t)=1-p_{i},
$$

where $F(t)$ is the standard normal distribution.

As shown in [26] an inequality of the form

$$
\pi\{X(\gamma) \leq 0\} \geq \alpha
$$

is equivalent to

$$
X_{\alpha}^{-} \leq 0,
$$

where $X_{\alpha}^{-}$is the left border of an $\alpha$-level set of $X$. So to transform the inequality (4) we need to find the left border of an $\alpha_{i}$-level set of the following fuzzy variable

$$
\mathrm{E}\left[f_{i}(x, \omega, \gamma)\right]-\beta_{i} \sqrt{\mathrm{D}\left[f_{i}(x, \omega, \gamma)\right]} .
$$

According to [22], the expectation of $f_{i}(x, \omega, \gamma)$ has the following possibilistic distribution

$$
\begin{aligned}
\mathrm{E}\left[f_{i}(x, \omega, \gamma)\right] & =\left[\mathrm{E}\left[m_{f_{i}}(x, \omega)\right], \mathrm{E}\left[d_{f_{i}}(x, \omega)\right]\right]_{L R}, \\
& =\left[m_{\mathrm{E}\left[f_{i}\right]}(x), d_{\mathrm{E}\left[f_{i}\right]}(x)\right]_{L R},
\end{aligned}
$$

where

$$
\begin{array}{r}
m_{\mathrm{E}\left[f_{i}\right]}(x)=\sum_{j=1}^{n}\left(\bar{a}_{i j}+\bar{\sigma}_{i j} m_{i j}\right) x_{j}-\left(\bar{a}_{i}+\bar{\sigma}_{i}\right) \\
d_{\mathrm{E}\left[f_{i}\right]}(x)=\mathrm{E}\left[\operatorname { m a x } \left\{\sigma_{i 1}(\omega) d_{i 1} x_{i 1}, \cdots,\right.\right. \\
\left.\left.\sigma_{i n}(\omega) d_{i n} x_{i n}, \sigma_{i}(\omega) d_{i},\right\}\right] .
\end{array}
$$

Here $\bar{a}_{i j}, \bar{a}_{i}$ and $\bar{\sigma}_{i j}, \bar{\sigma}_{i}$ are the mathematical expectations of $a_{i j}(\omega), a_{i}(\omega)$ and $\sigma_{i j}(\omega), \sigma_{i}(\omega)$, respectively.

In a similar way to the representation (3), the expectation (6) can be written as

$$
\mathrm{E}\left[f_{i}(x, \omega, \gamma)\right]=m_{\mathrm{E}\left[f_{i}\right]}(x)+d_{\mathrm{E}\left[f_{i}\right]}(x) \cdot Z^{0}(\gamma) .
$$

On the other hand, the variance can be represented as follows $[22]$

$$
\mathrm{D}\left[f_{i}(x, \omega, \gamma)\right]=\mathrm{E}\left[f_{i}(x, \omega, \gamma)-\mathrm{E}\left[f_{i}(x, \omega, \gamma)\right]\right]^{2} .
$$

Considering the from of $f_{i}(x, \omega, \gamma)$ and $\mathrm{E}\left[f_{i}(x, \omega, \gamma)\right]$, we can write that

$$
\begin{aligned}
& f_{i}(x, \omega, \gamma)-\mathrm{E}\left[f_{i}(x, \omega, \gamma)\right] \\
& =\left[m_{f_{i}}(x, \omega)-m_{\mathrm{E}\left[f_{i}\right]}(x), d_{f_{i}}(x, \omega)-d_{\mathrm{E}\left[f_{i}\right]}(x)\right]_{L R} \\
& =m_{f_{i}}(x, \omega)-m_{\mathrm{E}\left[f_{i}\right]}(x) \\
& \quad+\left(d_{f_{i}}(x, \omega)-d_{\mathrm{E}\left[f_{i}\right]}(x)\right) \cdot Z^{0}(\gamma),
\end{aligned}
$$

which leads to

$$
\begin{aligned}
\mathrm{D}\left[f_{i}(x, \omega, \gamma)\right]=\mathrm{E}[ & m_{f_{i}}(x, \omega)-m_{\mathrm{E}\left[f_{i}\right]}(x) \\
& \left.+\left(d_{f_{i}}(x, \omega)-d_{\mathrm{E}\left[f_{i}\right]}(x)\right) \cdot Z^{0}(\gamma)\right]^{2} .
\end{aligned}
$$

It is easy to see, that the expectation $\mathrm{E}\left[f_{i}(x, \omega, \gamma)\right]$ and the variance $\mathrm{D}\left[f_{i}(x, \omega, \gamma)\right]$ are functions of the fuzzy variable $Z^{0}(\gamma)$. Their $\alpha_{i}$-level sets can be found from the following lemma:

Lemma 1. If $X(\omega, \gamma)=f(Z(\gamma), a(\omega))$, where $a(\omega)$ is a random vector $a(\omega)=\left(a_{1}(\omega), \cdots, a_{k}(\omega)\right)$, then an $\alpha_{i}$-level set of the expected value $\mathrm{E}[X(\omega, \gamma)]$ is

$$
\mathrm{E}[X(\omega, \gamma)]_{\alpha}=\left\{\mathrm{E}[f(z, a(\omega))]: z \in Z_{\alpha}\right\},
$$

where $Z_{\alpha}$ is an $\alpha$-level set of $Z(\gamma)$.

Proof. The possibilistic distribution function of $X(\omega, \gamma)$ is

$$
\mu_{X}(\omega, t)=\sup _{z: \mathrm{E}[f(z, a(\omega))]=t} \mu_{Z}(z)
$$

Then an $\alpha$-level set will be

$$
\begin{aligned}
\mathrm{E}[X(\omega, \gamma)]_{\alpha} & =\left\{t: \sup _{z: \mathrm{E}[f(z, a(\omega))]=t} \mu_{Z}(z) \geq \alpha\right\} \\
& =\left\{t: \exists z, \mathrm{E}[f(z, a(\omega))]=t, \mu_{Z}(z) \geq \alpha\right\} \\
& =\left\{t: \exists z, \mathrm{E}[f(z, a(\omega))]=t, z \in Z_{\alpha}\right\} \\
& =\left\{\mathrm{E}[f(z, a(\omega))]: z \in Z_{\alpha}\right\} .
\end{aligned}
$$

And this completes the proof.

So, the left and the right borders of an $\alpha_{i}$-level set of $\mathrm{E}\left[f_{i}(x, \omega, \gamma)\right]$ will be $[4]$

$$
\begin{aligned}
& M_{i}^{-}(x)=m_{\mathrm{E}\left[f_{i}\right]}(x)-L^{-1}\left(\alpha_{i}\right) d_{\mathrm{E}\left[f_{i}\right]}(x), \\
& M_{i}^{+}(x)=m_{\mathrm{E}\left[f_{i}\right]}(x)+L^{-1}\left(\alpha_{i}\right) d_{\mathrm{E}\left[f_{i}\right]}(x),
\end{aligned}
$$

where $L^{-1}(t)$ is the inverse function of $L(t)$.

Considering the properties of a quadratic function, the borders of an $\alpha_{i}$-level set of $\mathrm{D}\left[f_{i}(x, \omega, \gamma)\right]$ will be $[2,7]$

$$
\begin{array}{r}
D_{i}^{-}(x)=\min \left\{\mathrm{E}\left[m_{D_{i}}(x, \omega)-L^{-1}\left(\alpha_{i}\right) d_{D_{i}}(x, \omega)\right]^{2},\right. \\
\left.\mathrm{E}\left[m_{D_{i}}(x, \omega)+L^{-1}\left(\alpha_{i}\right) d_{D_{i}}(x, \omega)\right]^{2}\right\}, \\
D_{i}^{+}(x)=\max \left\{\mathrm{E}\left[m_{D_{i}}(x, \omega)-L^{-1}\left(\alpha_{i}\right) d_{D_{i}}(x, \omega)\right]^{2},\right. \\
\left.\mathrm{E}\left[m_{D_{i}}(x, \omega)+L^{-1}\left(\alpha_{i}\right) d_{D_{i}}(x, \omega)\right]^{2}\right\},
\end{array}
$$

where

$$
\begin{aligned}
m_{D_{i}}(x, \omega) & =m_{f_{i}}(x, \omega)-m_{\mathrm{E}\left[f_{i}\right]}(x), \\
d_{D_{i}}(x, \omega) & =d_{f_{i}}(x, \omega)-d_{\mathrm{E}\left[f_{i}\right]}(x) .
\end{aligned}
$$


In the end, the borders of an $\alpha_{i}$-level set of (5) can be defined as $[17]$

$$
\left[M_{i}^{-}(x)-\beta_{i} \sqrt{D_{i}^{+}(x)}, M_{i}^{-}(x)-\beta_{i} \sqrt{D_{i}^{-}(x)}\right] .
$$

Now we are ready to formulate and prove the following theorems.

Theorem 1. Suppose that in the optimization model (1)-(2) fuzzy variables $b_{i j}(\omega, \gamma), b_{i}(\omega, \gamma)$ have the shiftscale representation, where fuzzy parameters $Z_{i j}(\gamma)$ and $Z_{i}(\gamma)$ are $T_{W}$-related and characterized by symmetrical distribution of LR-type; the shift and scale parameters are normally distributed random variables. Then the problem (1)-(2) is equivalent to the following stochastic model

$$
\begin{gathered}
M_{0}^{-}(x)-\beta_{0} \sqrt{D_{0}^{+}(x)} \rightarrow \min _{x}, \\
\left\{\begin{array}{l}
M_{i}^{-}(x)-\beta_{i} \sqrt{D_{i}^{+}(x)} \leq 0, i \in \overline{1, m} \\
x \in X,
\end{array}\right.
\end{gathered}
$$

where for $i=\overline{0, m}$

$$
\begin{gathered}
M_{i}^{-}(x)=m_{\mathrm{E}\left[f_{i}\right]}(x)-L^{-1}\left(\alpha_{i}\right) d_{\mathrm{E}\left[f_{i}\right]}(x), \\
D_{i}^{-}(x)=\min \left\{\mathrm{E}\left[m_{D_{i}}(x, \omega)-L^{-1}\left(\alpha_{i}\right) d_{D_{i}}(x, \omega)\right]^{2},\right. \\
\left.\mathrm{E}\left[m_{D_{i}}(x, \omega)+L^{-1}\left(\alpha_{i}\right) d_{D_{i}}(x, \omega)\right]^{2}\right\},
\end{gathered}
$$

$L^{-1}(t)$ is the inverse function of $L(t) ; \beta_{i}$ is the solution of the equation

$$
F(t)=1-p_{i},
$$

where $F(t)$ is the standard normal distribution function.

In the case when $p_{i}=0.5, i=\overline{0, m}$ the stochastic equivalents of the criteria function (1) and the constraints (2) can be simplified as stated in the following theorem.

Theorem 2. Suppose that in the optimization model (1)-(2) $p_{i}=0.5, i=\overline{0, m}$; fuzzy variables $b_{i j}(\omega, \gamma), b_{i}(\omega, \gamma)$ have the shift-scale representation, where fuzzy parameters $Z_{i j}(\gamma)$ and $Z_{i}(\gamma)$ are $T_{W}$ related and characterized by symmetrical distribution of LR-type; the shift and scale parameters are normally distributed random variables. Then the problem (1)-(2) is equivalent to the following stochastic model

$$
\begin{aligned}
& m_{\mathrm{E}\left[f_{0}\right]}(x)-L^{-1}\left(\alpha_{0}\right) d_{\mathrm{E}\left[f_{0}\right]}(x) \rightarrow \min _{x}, \\
& \left\{\begin{array}{l}
m_{\mathrm{E}\left[f_{i}\right]}(x)-L^{-1}\left(\alpha_{i}\right) d_{\mathrm{E}\left[f_{i}\right]}(x) \leq 0, i \in \overline{1, m} \\
x \in X
\end{array}\right.
\end{aligned}
$$

Proof. If probabilities $p_{i}=0.5, i=\overline{0, m}$, then $\beta_{i}=0$ and the square root components $\sqrt{D_{i}^{+}(x)}$ in the criteria function and in the constraints will vanish. So, considering the form of $M_{i}^{-}(x)$, we will have

$$
\begin{gathered}
m_{\mathrm{E}\left[f_{0}\right]}(x)-L^{-1}\left(\alpha_{0}\right) d_{\mathrm{E}\left[f_{0}\right]}(x) \rightarrow \min _{x}, \\
\left\{\begin{array}{l}
m_{\mathrm{E}\left[f_{i}\right]}(x)-L^{-1}\left(\alpha_{i}\right) d_{\mathrm{E}\left[f_{i}\right]}(x) \leq 0, i \in \overline{1, m}, \\
x \in X .
\end{array}\right.
\end{gathered}
$$

This concludes the proof.

In the case of $p_{i}=0.5$ the obtained stochastic model (9)-(10) is equivalent to the stochastic analogue of a possibilistic-probabilistic optimization problem in which the principle of the expected possibility is used to remove the uncertainty of the probabilistic type [19]:

$$
\begin{gathered}
k \rightarrow \min , \\
\pi\left\{\mathrm{E}\left[f_{0}(x, \omega, \gamma)\right] \leq k\right\} \geq \alpha_{0}, \\
\left\{\begin{array}{l}
\pi\left\{\mathrm{E}\left[f_{i}(x, \omega, \gamma)\right] \leq 0\right\} \geq \alpha_{i}, i=\overline{1, m} \\
x \in X
\end{array}\right.
\end{gathered}
$$

\section{Stochastic Quasi-Gradient Method}

The models (7)-(8) and (9)-(10) can be solved by deterministic techniques of mathematical programming. However, these techniques require the calculation of the precise values of the expected value and the variance of a random function.

In the case of the weakest t-norm, the expected value and the variance of a possibilistic-probabilistic function are of such a complex nature that the calculation of their precise values is almost impossible. Moreover, the results of this calculation are nonlinear nonconvex functions, which lead to non-convex optimization problems.

An alternative approach is to use the stochastic quasigradient (SQG) methods [6]. The SQG methods allow us to solve optimization problems with objective functions and constraints of such a complex nature that the calculation of the precise values of these functions (all the more of their derivatives) is impossible. The main idea of these methods consists of using statistical estimates for the values of the functions and of their derivatives instead of their precise values. The SQG methods generalize the well-known stochastic approximation methods for unconstrained optimization of the expectation of random functions.

The stochastic quasi-gradient method for solving the problem (9)-(10) has been described in $[4,23]$. 


\section{Conclusion}

In the present paper the problem of possibilisticprobabilistic optimization is studied. We consider that possibilistic-probabilistic variables have a shift-scale representation. In this representation random parameters are normally distributed, whereas fuzzy parameters are symmetrical variables of LR-type and are related by the weakest t-norm. Under these assumptions the stochastic equivalent of the original problem is obtained.

\section{References}

[1] F. Aiche, M. Abbas, D. Dubois, Chanceconstrained programming with fuzzy stochastic coefficients, Fuzzy Optim. Decis. Mak. 12(2) (2008) 125-152.

[2] G. Alefeld, J. Herzberger, Introduction to Interval Computations, Academic Press, New York, 1983.

[3] D. Dubois, H. Prade, Possibility Theory, Plenum Press, New York, 1988.

[4] Y. E. Egorova, A. V. Yazenin, The problem of posibilistic-probabilistic optimization, J. Comput. Syst. Sci. Int. 56(4) (2017) 642-667.

[5] Y. E. Egorova, A. V. Yazenin, A method for minimum risk portfolio optimization under hybrid uncertainty, J. Phys.: Conf. Ser. (2018) V. 973.012033.

[6] Y. M. Ermolev, Methods of Stochastic Programming, Nauka, Moscow, 1976 (in Russian).

[7] R. Fuller, T. Keresztfalvi, On generalization of nguyens theorem, Fuzzy Sets Syst. 41(3) (1991) $371-374$.

[8] D. H. Hong, Parameter estimations of mutually t-related fuzzy variables, Fuzzy Sets Syst. 123 (2001) 63-71.

[9] H. Katagiri, M. Sakawa, K. Kato, I. Nishizaki, Interactive multiobjective fuzzy random linear programming: Maximization of possibility and probability, Eur. J. Oper. Res. 188 (2008) 330-339.

[10] H. Kwakernaak, Fuzzy random variables i, Inf. Sciences 15 (1978) 1-29.

[11] H. Kwakernaak, Fuzzy random variables ii, Inf. Sciences 17 (1979) 253-278.

[12] M. K. Luhandjula, Linear programming under randomness and fuzziness, Fuzzy Sets Syst. 10(13) (1983) 45-55.
[13] M. K. Luhandjula, Fuzziness and randomness in an optimization framework, Fuzzy Sets Syst. $77(3)$ (1996) 291-297.

[14] M. K. Luhandjula, Optimisation under hybrid uncertainty, Fuzzy Sets Syst. 146 (2004) 187-203.

[15] M. K. Luhandjula, Fuzzy stochastic linear programming: Survey and future research directions, Eur. J. Oper. Res. 174 (2006) 1353-1367.

[16] S. Nahmias, Fuzzy variables in a random environment, in: M. M. Gupta, R. K. Ragade, R. R. Yager (Eds.), Advances in fuzzy sets theory and applications, NHCP, Amsterdam, 1979, pp. 165180.

[17] T. Nguen, E. A. Walker, A First Course in Fuzzy Logic, CRC Press, Boca Raton, 1997.

[18] M. L. Puri, D. A. Ralescu, Fuzzy random variables, J. Math. Anal. Appl. 114 (1986) 409-422.

[19] A. V. Yazenin, Linear programming with random fuzzy data, Soviet J. Comput. Syst. Sci. 30 (1992) 86-93.

[20] A. V. Yazenin, On a method of solving a problem of linear programming with random fuzzy data, J. Comput. Syst. Sci. Int. 36(5) (1997) 737-741.

[21] A. V. Yazenin, Possibilistic-probabilistic models and methods of portfolio optimization, Stud. Comp. Intel. 36 (2007) 241-59.

[22] A. V. Yazenin, Basic concepts of possibility theory: a mathematical apparatus for decisionmaking under hybrid uncertainty condition, Fizmatlit Publ., Moscow, 2016 (in Russian).

[23] A. V. Yazenin, Y. E. Egorova, On solution methods for tasks of possibilistic-probabilistic optimization, Vestnik TvGU. Seriya: Prikladnaya matematika 4 (2013) 85-103 (in Russian).

[24] A. V. Yazenin, I. S. Soldatenko, A portfolio of minimum risk in a hybrid uncertainty of a possibilistic-probabilistic type: comparative study, in: J. Kacprzyk, E. Szmidt, S. Zadrozny, K. T. Atanassov, M. Krawczak (Eds.), IWIFSGN/EUSFLA T-2017. AISC, Vol. 643, Springer, Cham, 2018, pp. 551-563.

[25] A. V. Yazenin, I. S. Soldatenko, On the problem of possibilistic-probabilistic optimization with constraints on possibility/probability, in: R. F. et al. (Ed.), WILF 2018, LNAI 11291, Springer, Switzerland, 2019, pp. 43-54.

[26] A. V. Yazenin, M. Wagenknecht, Possibilistic Optimization, Vol. 6/96, Brandenburgische Technische Universitat, Cottbus: Aktuelle Reihe, 1996. 